\title{
Examining cross-national research teamwork: revealing rewards and challenges
}

\author{
Lynn McAlpine \\ Department of Education, University of Oxford, Oxford, UK \\ Isabelle Skakni \\ Department of Educational Research, Lancaster University, Lancaster, UK \\ Anna Sala-Bubaré \\ Facultat de Psicologia, Ciències de l'Educació $i$ de l'Esport Blanquerna, \\ Universitat Ramón Llull, Barcelona, Spain \\ Crista Weise \\ Department of Basic, Developmental and Educational Psychology, \\ Universitat Autònoma de Barcelona, Barcelona, Spain, and \\ Kelsey Inouye \\ Department of Education, University of Oxford, Oxford, UK
}

\begin{abstract}
Purpose - Teamwork has long featured in social science research. Further, with research increasingly "crossnational," communication becomes more complex, for instance, involving different cultures, languages and modes of communication. Yet, studies examining team communicative processes that can facilitate or constrain collaboration are rare. As a cross-national European team representing varied disciplines, experiences, languages and ethnicities, we undertook to examine our communication processes with the aim to promote better qualitative research practices.

Design/methodology/approach - Viewing reflection as a tool for enhancing workplace practices, we undertook a structured reflection. We developed an empirically derived framework about team communication, then used it to analyse our interaction practices and their relative effectiveness.

Findings - The results highlighted two under-examined influences, the use of different modes of communication for different purposes and the need for face-to-face communication to address a particularly challenging aspect of research, negotiating a shared coding scheme to analyse diverse cultural and linguistic qualitative data.

Practical implications - The study offers a procedure and concepts that others could use to examine their team communication.

Originality/value - The communicative processes that can constrain and facilitate effective cross-national research team collaboration are rarely examined. The results emphasise the need for careful negotiations around language, epistemologies, cultures and goals from the moment collaboration begins in formulating a project, through applying for grant funds, to when the last paper is published - timely in a context in which such work is increasingly expected.
\end{abstract}

Keywords Teamwork, Research teams, Cross-national, Communication

Paper type Research paper

(C) Lynn McAlpine, Isabelle Skakni, Anna Sala-Bubaré, Crista Weise and Kelsey Inouye. Published by Emerald Publishing Limited. This article is published under the Creative Commons Attribution (CC BY 4.0) licence. Anyone may reproduce, distribute, translate and create derivative works of this article (for both commercial and non-commercial purposes), subject to full attribution to the original publication and authors. The full terms of this licence may be seen at http://creativecommons.org/licences/by/4.0/ legalcode

This work was supported by an European Erasmus + Programme under the project 'Researcher Identity Development: Strengthening Science in Society Strategies' [grant number 2017-1-ES01-KA203038303].

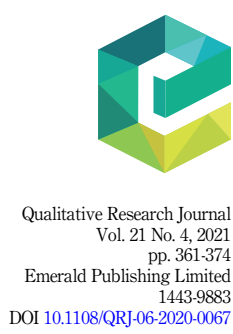


QRJ

21,4

362

\section{Context}

While teamwork has featured in research for some time, a recent global phenomenon is the funding expectation for research collaborations to be cross-national - a prime example being the policy and funding emphasis in the European Union (Lebeau and Papatsiba, 2016).

Studies of collaborative cross-national research have examined working with participants in collecting qualitative data (e.g. Kitchen, 2013; Martinus and Hedgcock, 2015). But, rarely have they explored the interaction of research teams (Tigges et al., 2019), particularly the communicative processes within which decisions are made (Kosmützkya, 2018; Gómez and Kuronen, 2001) - even though that have noted risks. Cultural (Payumo et al., 2019) and epistemic (Lebeau and Papatsiba, 2016) differences are potential barriers, as are inequalities across institutions (Leibowitz et al., 2014) and individuals' roles (Lebeau and Papatsiba, 2016). Of course, teams not perceiving the importance of communicative factors creates a risk (Brew et al., 2013) - and this may be common. For instance, principal investigators and researchers generally place greater emphasis on the methodological and task-related issues $(60 \%)$ than the interactive social complexities/ complications of diverse team collaboration $(40 \%)$ (Kosmützkya, 2018) [1].

Finally, the few studies addressing actual interaction processes included mixed methods (Curry et al., 2012); field-based studies (Easterby-Smith and Malina, 1999) and case study/ ethnography (Gómez and Kuronon, 2011). Notably, none examined the role of different modes of communication. Nor did they examine a particularly complex aspect of qualitative research, the analysis of data (Easterby-Smith and Malina, 1999), yet this tends to lack standardised procedures or ground rules to ensure quality (Miles and Huberman, 1994). Further, qualitative analysis is especially challenging when it is cross-linguistic (Wong and Poon, 2010).

\section{Goal}

To better understand our practices, we analysed our cross-national team interaction processes using a conceptual framework to structure our reflection (Leadbetter, 2008) and thus, advance our understanding of our workplaces practices (Tynjala, 2008). We focused on: (a) the influence of modes of communication on our interactions and (b) the negotiation of a shared coding scheme to analyse diverse cultural and linguistic data.

\section{Our team}

We are a cross-national EU-funded social science team of 15-20 members sharing a lingua franca, European English, using quantitative and qualitative methods to examine the experiences of $\mathrm{PhD}$ and post-PhD researchers. Our team began in 2014 and incorporates researchers from four countries, cultures and languages (Finland [2], French-speaking Switzerland, UK and Spain), in three time zones. Given our geographical distribution, we have depended on a range of virtual tools with occasional face-to-face meetings.

Our diverse prior life trajectories have powerfully influenced our present thinking and actions (McAlpine and Amundsen, 2018). We come with different social science expertise (psychology, sociology and education), epistemologies (socio-constructivism through critical realism) and years of experience (from Master's through to experienced PIs [3]). Since epistemologies and methods are culturally bound, meanings amongst team members may not be universally shared (Redmond, 2003). While nearly all are from the global north and nearly all female, we are diverse in ethnicity.

As our qualitative team research was inherently complex, we had already been using “auditing" processes (Guba and Lincoln, 1985) for rigour and consistency (Creswell, 2013), for instance, to ensure equivalence of data collection protocols across countries (Tyupa, 2011) and reduce potential misinterpretation from an ethnocentric perspective. So the data collection 
tools, a survey and interview, were developed by team discussion in English, then versions created for each country, which were then back-translated, before pilots in each country to assess appropriateness linguistically and culturally.

If asked, we would claim effectiveness in outcome measures, for instance, publishing extensively (co-authoring in groups of two or three), getting renewed funding and creating online resources based on research findings.

We undertook this structured reflection partway through our second grant after five of us [4] spent a week together to start qualitatively analysing parallel Spanish, Swiss and UK data sets [5] in order to co-publish a paper - a difficult task that foregrounded communication processes. The data we were analysing represented the significant experiences reported in free-write survey responses by both doctoral students and post-PhD researchers in the three countries. We worked as a whole group and in three sub-groups, one for each country. We represented Rambur's (2009) most complex, risky and least stable form of cross-national collaboration that demands individual willingness to invest in communicating and working together - and we had already done this over a number of years but not actually reflected on the strategies we had developed.

\section{Framing the research}

As studies of cross-national research team interaction processes have been largely overlooked (Kosmützkya, 2018), we searched in organisational and management studies to find research on team effectiveness in private sector projects [6], finding six concepts to frame our inquiry:

(1) Two first-order, relatively concrete, constructs provided the means to create a data display of team communication;

(2) Four second-order, more abstract, constructs were used to analyse the integrated results from the first-order constructs.

In elaborating these concepts, we have integrated both research team and private sector studies.

\section{First-order}

Modes of communication. Modes of communication represent a continuum from face-to-face through blended (face-to-face and virtual) to virtual-only interaction along three dimensions: (a) mix of modes; (b) degree of synchronicity, e.g. Skype and Zoom are closer to face-to-face than emails and electronic documents; and (c) value of exchanged information for achieving the project (Mesmer-Magnus et al., 2011). More information sharing occurs in teams using blended communication than in teams using face-to-face (or virtual) communication alone (Pinjani and Palvia, 2013) since different modes privilege different information sharing (Mesmer-Magnus et al., 2011). Face-to-face meetings are ideal for integrating both task and interpersonal interactions and dealing with broader strategic issues; Skype meetings tend to be more task-oriented, short and structured so members can report progress and set new deadlines, but issues can still be explored as they arise; emails focus more on tracking tasks that can be done independently. Finally, openness of information sharing (e.g. personal values) is reduced in virtual modes - likely due to the focus on task rather than relational interaction (Mesmer-Magnus et al., 2011).

Nature of the task. Nature of the task influences possible success along two dimensions: degree of (a) complexity and (b) required interdependence (interaction among team members), with complex tasks more difficult to achieve than simple tasks given greater demand for effective information sharing and limited opportunities for trust-building (Marlow et al., 2017) [7]. When combined with high virtuality, opportunities for misunderstandings and mistakes 
QRJ

21,4 increase. As well, required interdependence to complete the task can reduce potential success (Pinjani and Palvia, 2013). In other words, the less attention given to task complexity and interdependence, the greater potential difficulty.

\section{Second-order}

Diversity. Diversity in teams can lead to positive outcomes (Deeks, 2004) by increasing (a) creativity, (b) useful questioning, (c) range of perspectives and (d) knowledge. However, even in face-to-face teams, diversity can pose a challenge as regards differences in methods valued, the lack of a shared language (Curry et al., 2012), and degree of information sharing (MesmerMagnus et al., 2011). Being willing to perceive others as distinct from us - and assuming that team exchanges are likely not initially (fully) understood (Akkerman et al., 2006) are essential in using diversity effectively.

Type of diversity may influence information sharing and trust-building (Pinjani and Pinjani, 2013). Deep-level diversity represents idiosyncratic attitudes, values, preferences, whereas functional-level diversity represents more visible differences, including age, gender and work responsibilities. In highly virtual teams, deep-level diversity has a more significant positive relationship with the team process of information sharing and trust (positive confident expectation of others) than functional-level diversity. Thus, while functional-level diversity may be helpful in choosing a team, maintaining a team requires deep-level diversity.

Patterns of communication. Patterns of communication are represented in (a) frequency (builds trust, enhances quality and content exchange; in virtual teams helps reduce concern that individuals will not carry out their responsibilities) (Pinjani and Palvia, 2013); (b) quality (clarity, accuracy and completeness); (c) content (both task and relational communication are present) (Marlowet al.,2017); and(d) geographical distribution(CramptonandHinds, 2005). Regarding the last, with subgroups in different locations, tendency for tension may grow between the subgroups, unless subgroups value what they can learn from the qualities of the different groups.

Cultural sensitivity/competence. Cultural sensitivity/competence is directly linked to team diversity and can be understood as (a) a sensitivity to using one's knowledge and understanding to demonstrate respect towards others and (b) a willing self-awareness to tailor interactions with others (adapted from Foranda, 2008). The stance represents an effort to move away from ethnocentrism to communicate more effectively. The greater the team cultural diversity, the greater the need for cultural sensitivity (Crampton and Hinds, 2005). Positive attitudes towards cultural diversity increase project and innovation performance (Bouncken et al., 2008) especially important when there are sub-groups (Crampton and Hinds, 2005).

Trust. Trust is difficult construct to characterise, but is frequently linked to the nature of communication as well as individuals' prior trust experiences and institutional structures (Mach et al., 2010). Frequent communication, timely and predictable rather than sporadic, helps to build trust (Pinjani and Palvia, 2013). Still, diversity, while it can be positive, can also make trusting the "other" difficult, with a subsequent impact on handling conflicts and tensions (Curry et al., 2012). Further, virtual modes will reduce openness of information sharing which could negatively impact trust-building (Mesmer-Magnus et al., 2011). In other words, with increasing virtual communication, greater attention has to be given to trust-building. Overall, trust mediates the relationship between diversity and team effectiveness; it enhances team process, satisfaction and performance (Marlow et al., 2017; Pinjani and Palvia, 2013).

\section{Method}

We drew on the notion of reflection as a tool for enhancing workplace practices (e.g. Tynjala, 2008; Leadbetter, 2008) in doing a structured reflection on our group collaboration. The period spanned from November 2017, when the plan to meet was conceived, to March 2019, when the 
first paper was finalized. The first author synthesised the literature found. Then, using the two first-order constructs, we created a table to document our project (see final structure, Table 1), and populated it jointly by the evidence of our communication activities (diaries, research log books, code books, minutes of meetings, emails as well as documents stored on Google docs and other shared platforms).

This process of structuring our experience facilitated first-order reflection. We distinguished a range of communication modes:

(1) Two types of face-to-face:

- All members grant-funded: for planning, achieving and reporting goals;

- Non-grant funded: time added at conferences or workshops using non-grant funding;

(2) Two types of blended:

- Members funded on grant face-to-face; members not funded joining by Skype;

- Skype: all members on line but country teams usually in same room;

(3) Email exchanges: could be sub-set of team members (specific focus) or all members (team focus); number not tracked;

(4) Virtual document storage to facilitate team member access.

Further, the completed table helped us differentiate phases of greater and lesser complexity and degrees of interdependence. These were (a) designing the project; (b) becoming a team (extended face-to-face time); (c) advancing the project in country teams and full team; (d) first presentations which pushed us to interpretation; (e) furthering the project including reporting results (see Figure 1).

The final step in our collective reflection was for each of us, beginning with the first author, to read both the completed table and the framework and build a collective text - what you see in the results and discussion sections.

\section{Results}

First-order results: modes of communication and nature of the tasks

A-synchronous virtual meetings. Throughout the project, there was a constant stream of emails amongst all team and country team members. These concerned the accomplishment of
Examining cross-national research teamwork 


\section{QRJ \\ 21,4}

\section{6}

Figure 1.

Project phases and timeline

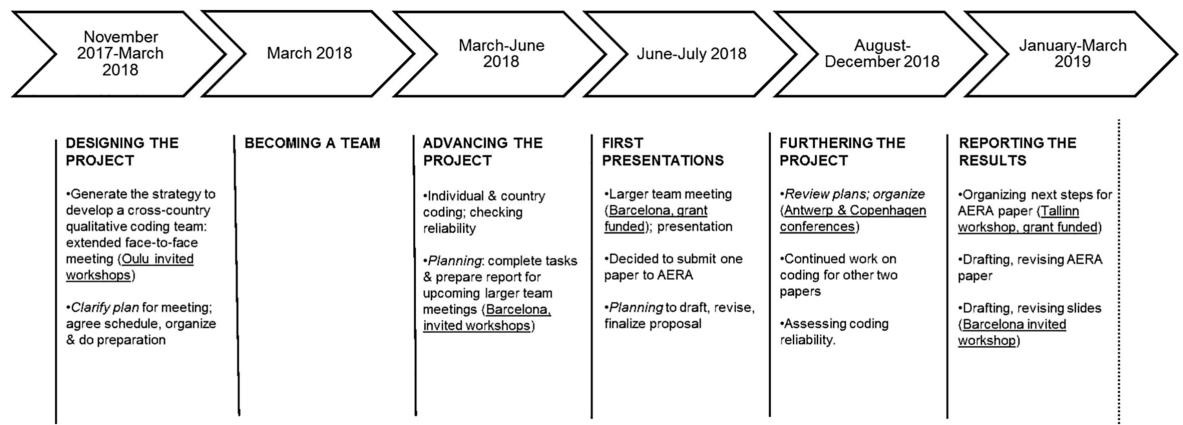

individual tasks and the need to postpone deadlines. All documents were also stored virtually on an ongoing basis for easy access. We quickly learned we needed conventions, including date and version stamping as our archive grew. Thus, an important aspect of virtual communication modes featured the rather simple technological devices we used as a team to work together: e-mail and Skype to communicate, and the same qualitative software (MaxQDA) to analyse data.

An example: Before our first face-to-face meeting, we had a Skype meeting, and used emails, virtual documents and a common virtual storage site to be sure that when we met we could begin relatively quickly the actual work of developing a cross-country qualitative codebook for analysing our data. This included organising and importing the different country data sets in a consistent fashion into qualitative software, doing background reading and for several learning to use the analysis software.

Face-to-face meetings. There were eight face-to-face meetings of more than a day (underlined in Figure 1), only two funded by the grant [8]. Face-to-face meetings were crucial during the analysis process. Collectively analysing qualitative data sets from different countries, a highly complex task, would have been largely impossible through any virtual mode of communication, especially initially. In two cases, we supplemented the face-to-face meetings by Skype so that a missing member could join. Another element of each phase that ensured the project progressed was time set aside to assess progress and plan forward (italicised in Figure 1).

Virtual synchronous meetings. We had nine Skype calls where we did similar tasks to faceto-face meetings, but in less depth, for instance, discussing refinements to code definitions as coding progressed. These meetings usually involved all authors, with actual coding completed independently beforehand. While we were task focused, we usually began our discussion with a relational focus: a round-robin update that helped maintain relationships. The three country teams similarly had face-to-face meetings, and communicated virtually through email exchange.

Second-order results: diversity, communication processes, cultural sensitivity and trust Here we examine the intersection of diversity, communication processes, cultural sensitivity and trust, referring to the literature as appropriate. As noted earlier, our task was complex and required the interdependence of its members: negotiating code definitions in three different languages, consistent within- and across-country coding and subsequent crosscountry analysis and interpretation of findings. First, we overview our reflections, offering examples; and then focus on practice in the our week together in March 2018 since it provides the richest experience to draw on. 
We would argue that the degree of blended communication we created - perhaps not as intentionally as we might - provided a positive and effective [9] team environment. Overall, we had frequent interactions at the end of which we set action items and deadlines; we had recourse to our records to enhance clarity; and we had opportunities for both social and task interactions. These factors influence trust-building, with trust mediating diversity (MesmerMagnus et al., 2011) and effective information-sharing (Marlow et al., 2017).

Our working assumption for face-to-face meetings was that they were likely to provide the most quality as regards negotiating the complexities of the task by facilitating openness of information sharing. These meetings largely focused on jointly conceptualising the work, negotiating shared understandings of the cross-linguistic coding (Wong and Poon, 2010) and exploring our collective thinking - and often included lunch or dinner together where there was more exchange on a personal level - an aspect of trust-building limited in virtual communication (Marlow et al., 2017). In retrospect, we realise that tracking the social opportunities of our experience would have been helpful since they were informal occasions where we learned individuals' biographies and hopes - and thus created greater bonds of trust in negotiating the task itself.

In our mind, this suggests that the team perceived the value of face-to-face communication, especially in dealing with complex tasks, developing a more intimate knowledge of other team members and building trust (Marlow et al., 2017; Pinjani and Palvia, 2013). We also believe that having these full-team meetings mitigated whatever tendency might have existed for tension to grow between the subgroups working in different locations (Crampton and Hinds, 2005). Overall, while sometimes being arduous and requiring patience, working together face-to-face was highly engaging and stimulating. So, for instance, our coding discussions led us to talk about how different our countries were in the way people communicate, and how this could make for tricky communication and relationships - and this was one of the stimuli for this paper.

As noted earlier, we represented ethnic, cultural, linguistic and disciplinary diversity, and ranged from $\mathrm{PhD}$ student through to semi-retired professor. The influence of this deep-level diversity (Pinjani and Palvia, 2013) on our interactions is more difficult to document and describe. One concrete example would be that we had to "unpack" our taken-for-granted disciplinary assumptions (Redmond, 2003) about sense-making and emotions which were central to the analysis, yet are viewed differently in sociology and psychology. As well, we all shared the experience of choosing to live in countries other than our own, so it is also likely we held positive attitudes towards and a high level of cultural sensitivity that would help increase project effectiveness (Bouncken et al., 2008). For instance, we would often make jokes about our own cultures. Finally, we shared a commitment to enhancing the support of early career researchers and conducting highly rigorous research (Shulha and Wilson, 2003), which likely helped us deal with the frustrations of the difficult negotiations we experienced.

Nevertheless, the task we faced in our one-week meeting was a daunting one: to negotiate distinct individual trajectories of experience, discipline, epistemology, culture and language as we worked to develop a common understanding and language for our work together (Wong and Poon, 2010). That is, our task was to negotiate (and use) this deep-level diversity (Pinjani and Palvia, 2013) in order to build trust and progress towards our goal, which meant remaining productive despite the work being both exhausting and exhilarating. We try below to give a sense of this.

Our goal for the end of the week was to have sufficiently established codes and definitions that we could continue our work individually. Each day began with the team reviewing the agreed agenda (set at the end of the previous day), as well as the notes recorded on flip chart and whiteboard. The team leader proposed timing for each activity, tracked progress and suggested "parking" discussions when we were "stumped" until we came across other segments that were similar. We took breaks and lunch when the team felt 
QRJ

21,4

368

ready for a break. These moments of informal exchange at the university allowed us to revisit the examples we were analysing in an embedded way related to our own personal experiences and perspectives.

As to the actual analysis, first, all five jointly coded some English data, which consisted broadly in defining the recurrent themes that emerged from the respondents' responses. The aim was to develop English code names and definitions and build a shared codebook. Then, still using English data, we individually coded the same data, using our preliminary codes and discussed the results. Finally, country teams jointly coded country data and then we discussed issues across the team.

In practice, in generating a coding procedure, we had to negotiate the wording of the code name and its definition in English using English data. This English discussion had to take into consideration how these names and definitions might "work" in French and Spanish, given different cultural/linguistic ways of conceiving and representing the world. Generally, this involved country teams translating data from French or Spanish into English in order to discuss interview excerpts with the whole group. Yet, this approach could be problematic when specific idioms were impossible to translate directly into English. In other cases, the same English word could hold different meaning depending on the language, which induced discussions about the most accurate representations of the concepts we were trying to code. Thus, at any sign of disagreement or lack of coding consistency across languages, we took a step back and tried to make our thoughts, definitions and assumptions explicit. A concrete example of this was the difficulty we had in coding "accomplishment" consistently across country data. Through careful deliberation, we found that the source of the difficulty was that, while in both languages the word has two meanings, each team was focusing on only one meaning - and not the one the other team was using. So, we explicitly defined the code as including both, which resolved our consistency problem. As can be imagined, this process was not straightforward, as we stopped frequently to discuss these emerging issues.

When issues were resolved relatively quickly through discussion, they were recorded electronically in code memos in MaxQDA and in a Word document summarising protocols for the coding and verification process. When issues appeared difficult to resolve, that is, we could not find a way forward after five-eight minutes of discussion, we posted the issue (with notes) on a whiteboard to return to later. We viewed these notes as visual prompts to look for examples in the different data sets that might contribute to resolution of the difficulty. We regularly returned to these at least once a day. Further, as we progressed, we became aware of slight variances in the different data sets that might influence the specific wording of the research questions, so we tracked these issues visually as well, but on a flip chart [10] - again reviewing and making changes as needed.

Once we had established consistency in the English data set, the country teams coded country data assessing the usability of the codes developed in English. As issues arose, we discussed them in the whole team. For instance, in each country data set, participants included those who used the national language as a lingua franca and this too required understanding and careful interpretation. We trusted the country teams to work together to carefully negotiate joint interpretations (Wong and Poon, 2010) that would later inform our overall study - and calculated reliability to make sure the interpretations were not biased.

Challenges such as the ones noted above, while sometimes frustrating in the moment, were usually resolved, creating a rewarding sense of accomplishment (Neumann, 2006). Overall, such experiences strengthened the analysis procedure as well as the bond of mutual trust as we came to understand better our distinct practices. In other words, this meeting, the start of our joint enterprise, was an intense (and intensive) social and scholarly experience that created the relationships on which to build our later largely virtual communication.

Overall, we advanced the project using blended modes of communication, taking advantage of the benefits of each in an integrated fashion: ensuring the amount and type of 
information shared (Pinjani and Palvia, 2013). Face-to-face meetings focused on tasks that required the most interdependence, interaction and synchronicity, that is, processes that embodied and required trust-building such as deciding the objectives of the study, developing and discussing the codebook. Virtual communication (mainly exchange of information and documents) increased before face-to-face meetings to ensure we made the most of our time together. Such communications were flexible and adapted to our schedules and calendars, allowing individuals to stay connected and work on the project at their convenience. Finally, our diverse backgrounds offered more perspectives when discussing and interpreting the results. As Gómez and Kuronen (2011) note, such discussions are fruitful since they open up to inspection theoretical, methodological and practical distinctions. This is true regardless of the role or status of a team member [11].

\section{Discussion}

We analysed our cross-national team interaction processes through a structured collaborative reflection to better understand and enhance our teamwork. While this process helped us, the scarcity of studies on cross-national research team interaction processes raises concerns in a context in which such work is increasingly expected. We begin by discussing the contributions of our study and then the broader issues for cross-national research generally.

\section{Applying the framework}

This is the first report we know that explored cross-national researchers' interaction processes and incorporated modes of communication - which we believe is central to understanding such inquiry, yet not often done (Gómez and Kuronen, 2001). Using a structured collaborative reflection enabled us to examine closely our communicative interaction processes. Further, we examined a particularly challenging aspect of such communication: the negotiation of a coding scheme to analyse diverse cultural and linguistic qualitative data. We suggest the process including the conceptual framework provide a structure for others - and refer you as well to the Cochrane Collaboration's guidelines (Deeks, 2004) and Curry et al.'s (2012) set of principles.

Our focus was on coding culturally and linguistically distinct data so embodying many critical issues about team communication. Overall, face-to-face communication significantly helped us negotiate cultural, linguistic, disciplinary and other forms of diversity, and through this, sometimes challenging process, build trust. Using European English as a lingua franca enabled us to negotiate amongst ourselves, but negotiating was also an arduous and timeconsuming task that required patience and careful listening. In this regard, fluency in the lingua franca provides power in discussions, so a researcher's lack of fluency might reduce desire and ability to participate in such research. For this reason, in choosing the team members, we had been attentive to this characteristic.

As well, use of a lingua franca, particularly as regards coding, may preclude alternate interpretations emerging through other language and cultural lenses - and might also be true in within-country coding where local and/or regional distinctions may become lost. Such factors have the potential to result in distorted interpretations. In that sense, this teamwork experience led us to become more aware of our own cultural, personal and disciplinary biases that have enriched our way of analysing and interpreting qualitative data - and our future work together.

Notably, trust between team members is not necessarily what you already bring to the process (Lebeau and Papatsiba, 2016), but one that can be built (or not) through the process while recognising that individuals bring their own trust experiences to the interaction (Mach et al., 2010). Recall that before the week-long face-to-face meeting we did not all know each other well. Thus, we would argue that recognising and explicitly addressing disparate
Examining cross-national research teamwork 
QRJ

21,4

differences, which teams do not always do (Brew et al., 2013), makes it easier to deal with the complexity of any research undertaking. In our case, trust took the form of a recognition of and respect for each team member's approaches, skills and style of work.

The analysis suggested we had created a blended pattern of communication that enabled more meaningful information-sharing than face-to-face or virtual alone. This pattern emerged without forethought rather than from the evidence-based perspective provided by the conceptual framework. Now, going forward, we have a framework for ensuring explicit attention to this issue.

Further, the initial investment in extended face-to-face interactions to develop the coding scheme was essential for later successful virtual communication. Still, we would not have been able to advance our work without the many virtual exchanges of information about the project, such as tracking progress and negotiating deadlines. In other words, our modes of communication helped us to strike a balance between meeting the agreed timeline and respecting team members' work style and availability. Still, a limitation is that we were not as intentional as we might have been in planning our blend of modes of communication, so may not have been as effective in our communication as we could have been.

Finally, while the analysis led us to more explicitly name the nature and effectiveness of this approach, as well as some challenges, we do not recall serious frictions and misunderstandings - a limitation of doing the analysis retrospectively. We need in future interactions to be attentive to such frictions that might at first glance appear miniscule but could imply deep differences. However, our overall experience highlights several teamwork competencies that will be increasingly in demand in the current global research world.

\section{Limitations and future research}

As noted earlier, the lack of research on cross-national research teams led us to the organisational and management literature where interaction processes of project management have been studied. Future research might explore other potentially relevant literatures.

Further, while we intentionally did not address the interaction of individual factors with structural factors such as university provision of virtual resources, previous studies on research collaboration have demonstrated the influence of both structural and individual factors, past and present, on success - and that the two intersect (Brew et al., 2013). Key here is that structural supports are necessary but insufficient for success. So, more research is needed. As well, the analysis by Rambur (2009) highlights that research collaborations exist as a continuum from simpler to more complex. Future research could examine how the variation in degree of complexity interacts with the use of different modes of communication. We also concur with Marlow et al. (2017) that we need to break down even further the ways in which different virtual modes and blends of modes play a role. Overall, future research needs to pay more attention to the complexity of communicative interaction processes. This is especially important since qualitative social science analysis often appears individually driven (Redmond, 2003), but this cannot - should not - be the case in cross-national team analysis. Finally, given the COVID-19 pandemic has led us to near total virtual team communication, the framework can serve to remind of what we may be missing in our communication processes.

\section{Conclusion}

What lessons do we draw from this analysis? First, the two-step process of reflective analysis enabled an assessment of the team's task: negotiating a coding scheme for diverse cultural and linguistic qualitative data. The first-order constructs allowed us to document the nature of our cross-national teamwork; and the resulting data display enabled an analysis of the 
subjective aspects of the process as well as of our effectiveness using the second-order constructs.

The empirical framework helped us break down and reflect on the complexity of our crossnational project, particularly as regards individual efforts to negotiate difference and advance both the project and team cohesion. We believe it offers a relatively robust means to examine any collaborative research teamwork since it provides a language for team reflexivity that helps reveal the taken-for-granted.
Examining cross-national research teamwork

\section{Key points}

(1) This paper focuses on issues faced by cross-national research teams, focusing on a relatively under-researched line of inquiry, influence of different modes of communication.

(2) It offers a unique perspective in focusing on the interaction processes of team interaction in a particularly challenging aspect of research - negotiating a shared coding scheme to analyse diverse cultural and linguistic qualitative data.

(3) It offers evidence-based reflective tools and a procedure for other cross-national researchers to analyse the nature and effectiveness of their own teamwork - timely in a context in which such work is increasingly expected, especially given the dramatic shift to nearly complete virtual communication since the arrival of the pandemic.

\section{Notes}

1. Most of these studies represent cross-cultural work in the Global North.

2. A Finnish colleague, $(\mathrm{NN})$, joined us initially as preparation for future similar data collection and analysis in Finland.

3. Thus, some team members are engaged in research related to their lived experience.

4. We represent much the same diversity as the larger team, though we had all lived and worked in other countries, which may not be true of all in the larger team.

5. For more about our work, see https://www.researcher-identity.com/

6. Our interpretation of these may not be the original ones given they emerge from private sector studies.

7. This characterisation of complexity focuses on communicative interaction not the task as a whole, thus breaking down what complexity means in Rambur's (2009) framework.

8. We managed this by taking advantage of conferences where more than one team member was present.

9. From this work together, there has been one conference paper and two journal articles.

10. This was to differentiate visually the nature of the concern: whiteboard ephemeral micro issues, flip chart ongoing core issues.

11. We were fortunate perhaps in not dealing with national teams with considerable cultural distance, i.e. UK and Chinese, as in Easterby-Smith and Malina (1999).

\section{References}

Akkerman, S., Admiraal, W., Simons, R. and Niessen, T. (2006), "Considering diversity: multivoicedness in international academic collaboration”, Culture and Psychology, Vol. 12 No. 4, pp. 461-485. 
QRJ

21,4

Bouncken, R., Ratzmann, M. and Winkler, V. (2008), "Cross-cultural innovation teams: effects of four types of attitudes towards diversity", Journal of International Business Strategy, Vol. 8 No. 2, pp. 26-36.

Brew, A., Boud, D., Lucas, L. and Crawford, K. (2013), "Reflexive deliberation in international research collaboration: minimising risk and maximising opportunity", Higher Education, Vol. 66 No. 1, pp. 93-104.

Crampton, C. and Hinds, P. (2005), "Subgroup dynamics in internationally distributed teams: ethnocentrism or cross-national learning?", Research in Organisational Behaviour, Vol. 26, pp. 231-263.

Creswell, J. (2013), Qualitative Inquiry and Research Design: Choosing Among Five Approaches, Sage, Thousand Oaks.

Curry, L., O'Cathain, A., Plano Clark, V., Aroni, R., Fetters, M. and Berg, D. (2012), "The role of group dynamics in mixed methods health sciences research teams", Journal of Mixed Methods Research, Vol. 6 No. 1, pp. 5-20.

Deeks, M. (2004), "Cross-cultural team working within the Cochrane collaboration”, Downloaded March 2, 2019, available at: https://training.cochrane.org/sites/training.cochrane.org/files/ public/uploads/resources/downloadable_resources/English/crossculturalteamwork_000.pdf.

Easterby-Smith, M. and Malina, D. (1999), "Cross-cultural collaborative research: towards reflexivity", The Academy of Management Journal, Vol. 42 No. 1, pp. 76-86.

Foranda, C. (2008), "Cultural sensitivity: a concept analysis", Journal of Transcultural Nursing, Vol. 19 No. 3, pp. 207-212.

Gómez, M.V. and Kuronen, M. (2011), "Comparing local strategies and practices: recollections from two qualitative cross-national research projects", Qualitative Research, Vol. 11 No. 6, pp. 683-697.

Guba, E. and Lincoln, Y. (1985), Naturalistic Inquiry, Sage, Beverly Hills, US.

Kitchen, M. (2013), "Methods in focus group interviews in cross-cultural settings: empowering migrant voices", Qualitative Research Journal, Vol. 13 No. 3, doi: 10.1108/QRJ-01-2013-0005.

Kosmützkya, A. (2018), "International team research in comparative higher education: shedding some light on its social side", Journal of Comparative and International Higher Education, Vol. 10, pp. 14-23.

Leadbetter, J. (2008), "Learning in and for interagency working: making links between practice development and structured reflection", Learning in Health and Social Care, Vol. 7 No. 4, pp. 198-208.

Lebeau, Y. and Papatsiba, V. (2016), "Conceptions and expectations of research collaboration in the European social sciences: research policies, institutional contexts and the autonomy of the scientific field”, European Educational Research Journal, Vol. 15 No. 4, pp. 377-394.

Leibowitz, B., Ndebele, C. and Winberg, C. (2014), “'It's an amazing learning curve to be part of the project": exploring academic identity in collaborative research", Studies in Higher Education, Vol. 39 No. 7 , pp. 1256-1269.

Mach, M., Dolan, S. and Tzafrir, S. (2010), "The differential effect of team members' trust on team performance: the mediation role of team cohesion", Journal of Occupational and Organizational Psychology, Vol. 83, pp. 771-794.

Marlow, S., Lacerenza, C. and Salas, E. (2017), "Communication in virtual teams: a conceptual framework and research agenda", Human Resources Management Review, Vol. 27, pp. 575-589.

Martinus, K. and Hedgcock, D. (2015), "The methodological challenge of cross-national qualitative research: comparative case study interviews in Australia and Japan”, Qualitative Research Journal, Vol. 15 No. 3, pp. 373-386.

McAlpine, L. and Amundsen, C. (2018), Identity-trajectories: Ways of understanding post-PhD career choices, Palgrave MacMillan, Basingstoke. 
Mesmer-Magnus, J., DeChurch, L., Jimenez-Rodriguez, M., Wildman, J. and Shuffler, M. (2011), “A meta-analytic investigation of virtuality and information sharing in teams", Organisational Behaviour and Human Decision Processes, Vol. 94 No. 2, pp. 535-546.

Miles, M. and Huberman, A. (1994), Qualitative Data Analysis, Sage, Beverly Hills.

Neumann, A. (2006), "Professing passion: emotion in scholarship of professors at research universities", American Educational Research Journal, Vol. 43 No. 3, pp. 381-424.

Payumo, J., Moore, D., Evans, M. and Arasu, P. (2019), "An evaluation of researcher motivations and productivity outcomes in international collaboration and partnerships at a US researchintensive university”, Interdisciplinary Journal of Partnership Studies, Vol. 6 No. 2, p. 4, available at: pubs.lib.umn.edu/ijps.

Pinjani, P. and Palvia, P. (2013), "Trust and knowledge sharing in diverse global virtual teams", Information and Management, Vol. 50, pp. 144-153.

Rambur, B. (2009), "Creating collaboration: an exploration of multinational research partnerships", in Brew, A. and Lucas, L. (Eds), Academic Research and Researchers, Society for Research into Higher Education and the Open University Press, Basingstoke, pp. 80-95.

Redmond, M. (2003), "Cultural and ethical challenges in cross-national research: reflections on a European Union study on child and youth migration”, International Journal of Qualitative Methods, Vol. 2 No. 4, pp. 15-24.

Shulha, L. and Wilson, R. (2003), "Collaborative mixed methods research", in Tashakkori, A. and Teddlie, C. (Eds), Handbook of Mixed Methods in Social and Behavioral Research, Sage, Thousand Oaks, pp. 639-670.

Tigges, B., Miller, D., Dudding, K., Balls-Berry, J., Borawski, E., Dave, G., Hafer, N., Kimminau, K., Kost, R., Littlefield, K., Shannon, J. and Menon, U. (2019), "Measuring quality and outcomes of research collaborations: an integrative review", Journal of Clinical and Translational Science, Vol. 3, pp. 261-289.

Tynjala, P. (2008), "Perspectives into learning at the workplace", Educational Research Review, Vol. 3, pp. $130-154$.

Tyupa, S. (2011), “A theoretical framework for back -translation as a quality assessment tool”, New Voices in Translation Studies, Vol. 7, pp. 35-46.

Wong, J. and Poon, M. (2010), "Bringing translation out of the shadows: translation as an issue of methodological significance in cross-cultural qualitative research", Journal of Transcultural Nursing, Vol. 21 No. 2, pp. 151-158.

\section{About the authors}

Lynn McAlpine, I am an English first-language female who worked for 20 years as a professional before becoming an academic. As an academic, I have been privileged to work cross-culturally in the Canadian Arctic and sub-Arctic, Indonesia, Sri Lanka and Africa. And, while I began my academic life in Canada, I now live largely in the UK. I have been involved in a number of different cross-national research teams since 2006.

Isabelle Skakni, I am a French first-language North American female in my fifth post-PhD year. After receiving my $\mathrm{PhD}$ from a Canadian university, I moved to Europe for a postdoctoral fellowship. In addition to being affiliated to a university in the UK, I am now head of the doctoral training office at a Swiss university. My expertise is in the field of early career researchers' experiences and development. I have been involved in cross-national projects since 2016.

Anna Sala-Bubaré, I am a Catalan first-language South-European female in my second post-PhD year. My research deals with researchers' education and development, with a special focus on academic writing processes. During my studies, I lived in four European and American countries in Europe and America. I have been involved in cross-national research projects since 2012.

Crista Weise, I am a Spanish first-language South American female living in Europe since 2007. Before receiving my PhD from a Spanish University I lived, worked and researched for around 15 years in various South American countries, both at university and as a professional. During these years I participated in cross-culturally projects involving American indigenous people, Asian and European 
teams. My research focus is in higher and elementary education. Crista Weise is the corresponding author and can be contacted at: crista.weise@uab.cat

Kelsey Inouye, I am an English first-language female from the United States, currently in the third year of a $\mathrm{PhD}$ in the UK. My research focuses on doctoral writing and researcher development. Prior to the $\mathrm{PhD}$, I attended college and law school in Hawaii. This was my first experience working in a cross-national research team.

For instructions on how to order reprints of this article, please visit our website: www.emeraldgrouppublishing.com/licensing/reprints.htm Or contact us for further details: permissions@emeraldinsight.com 